\title{
DISSIPATIVE DIVERGENCE OF RESONANT ORBITS
}

\author{
Konstantin Batygin ${ }^{1,2}$ and Alessandro Morbidelli ${ }^{3}$ \\ ${ }^{1}$ Division of Geological and Planetary Sciences, California Institute of Technology, Pasadena, CA 91125, USA \\ ${ }^{2}$ Institute for Theory and Computation, Harvard-Smithsonian Center for Astrophysics, 60 Garden St., Cambridge, MA 02138 \\ ${ }^{3}$ Departement Cassiopée, Universite de Nice-Sophia Antipolis, Observatoire de la Côte d'Azur, F-06304 Nice, France; kbatygin@gps.caltech.edu \\ Received 2012 April 12; accepted 2012 July 19; published 2012 November 20
}

\begin{abstract}
A considerable fraction of multi-planet systems discovered by the observational surveys of extrasolar planets reside in mild proximity to first-order mean-motion resonances. However, the relative remoteness of such systems from nominal resonant period ratios (e.g., 2:1, 3:2, and 4:3) has been interpreted as evidence for lack of resonant interactions. Here, we show that a slow divergence away from exact commensurability is a natural outcome of dissipative evolution and demonstrate that libration of critical angles can be maintained tens of percent away from nominal resonance. We construct an analytical theory for the long-term dynamical evolution of dissipated resonant planetary pairs and confirm our calculations numerically. Collectively, our results suggest that a significant fraction of the near-commensurate extrasolar planets are in fact resonant and have undergone significant dissipative evolution.
\end{abstract}

Key words: methods: analytical - methods: numerical - planets and satellites: general

Online-only material: color figures

\section{INTRODUCTION}

Among the most unexpected discoveries brought forth by extrasolar planetary surveys to date has been the identification of numerous planetary bodies that reside in close proximity to their host stars. Planets of this sort are of great scientific interest because they represent a class of objects unavailable for study in our own solar system. In turn, observational characterization of such planetary systems can yield avenues toward identifying specific physical/dynamical behavior that does not occur locally, thus broadening our knowledge of the possible evolutions of planetary systems.

A readily apparent dynamical feature of close-in extrasolar planetary systems, highlighted by observational surveys such as the Kepler mission (Borucki et al. 2011; Lissauer et al. 2011), is the prominence of near mean-motion commensurabilities (i.e., integer period ratios) among sub-giant planets (Figure 1). Accordingly, understanding how close-in planetary systems attain near-resonant orbital architectures is the primary focus of this work.

The process of resonant locking requires slow, convergent orbital evolution of planetary bodies (Goldreich 1965; Peale 1976). It is likely that torques associated with disk-driven migration often lead to resonant coupling, and it has been suggested that near-exact commensurability should be maintained as planets travel through their protoplanetary disks (Terquem \& Papaloizou 2007; Cresswell \& Nelson 2008). However, the onset of magnetorotational instability (Balbus \& Hawley 1991) and the associated turbulence in protoplanetary disks can act to disrupt mean-motion resonances (Adams et al. 2008; Rein \& Papaloizou 2009; Ketchum et al. 2011). Thus, if disks are violently turbulent, resonant objects should be rare.

As already hinted above, the observations show that there exists a characteristic regime in between the two extremes, and the precise dynamical nature of this regime is elusive. Particularly, planets often reside sufficiently far away (a few percent or more) from their nominal first-order resonant locations (i.e., period ratios of $2: 1,3: 2$, and $4: 3$ ) to be readily interpreted as nonresonant. Yet the preference for orbits just wide of resonance and a characteristic pile-up of near-resonant objects (Figure 1) is suggestive of a common evolutionary path. Indeed, the mechanism responsible for such configurations has been noted to be a subject of great theoretical interest (Fabrycky et al. 2012).

It is possible in principle that most sub-giant planets arrive onto their close-in orbits in resonance and subsequently diverge away from exact commensurability due to tidal dissipation. Tides alone affect the semimajor axes only on very long timescales (often much longer than the Hubble time). However, as shown by the nonlinear perturbative calculations and $N$-body simulations aimed at reproducing the orbital configurations of the HD40307 (Papaloizou \& Terquem 2010) as well as GL581 and HD10180 (Papaloizou 2011) systems, resonant interactions can be quite effective at converting tidal eccentricity damping (which acts much faster) into a divergence of the orbital semimajor axes of the resonant bodies. In particular, the said simulations suggest that resonant coupling can be maintained far from nominal resonant locations and significantly aids in enhancing orbital divergence.

The calculations performed by Papaloizou \& Terquem (2010) motivate our development of a general qualitative understanding of the orbital evolution of close-in resonant planetary systems subject to dissipative effects. Thus, the development of an analytical theory for dissipative divergence of resonant orbits is the primary focus of this paper. The number of wellcharacterized systems within the Kepler sample remains limited and estimation of planetary masses from radii alone is generally risky (Stevenson 1982; Rogers et al. 2011). Consequently, in this work, we shall concentrate our efforts on characterization of the physical process rather than reproduction of any particular orbital architecture. Still, we argue that the interplay between resonant effects and tidal dissipation is the primary mechanism by which planets attain near-commensurate orbits. Lithwick \& $\mathrm{Wu}$ (2012) arrived at many of the results presented in this work simultaneously and independently; their paper was posted on arxiv.org at the same time as this one.

The paper is organized as follows. In Section 2, we set the stage by developing an integrable approximation to the conservative dynamics of a resonant pair at low eccentricities 


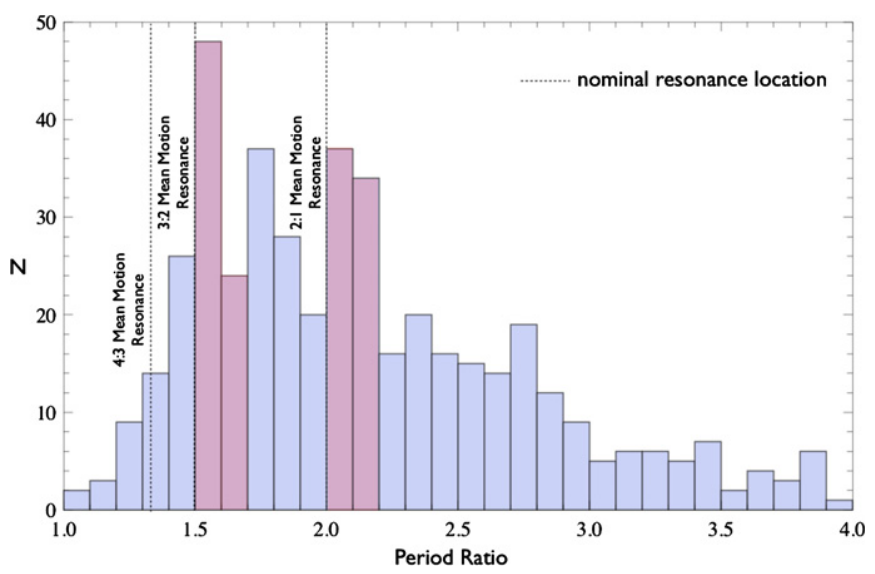

Figure 1. Histogram of the period ratios of all planet pairs detected by the Kepler mission with no filters on planetary radius or orbital period (http://planetquest.jpl.nasa.gov/kepler). In systems where more than two planets are present, only the neighboring period ratios are reported. Note the highlighted enhancement of objects immediately outside of the common $(2: 1$ and $3: 2)$ firstorder mean-motion resonances.

(A color version of this figure is available in the online journal.)

and validate the theory by comparison with $N$-body simulations. In Section 3, we introduce dissipation into the problem and show that tidal effects drive the system toward a quasi-stationary state that is characterized by an irreversible drift away from nominal resonance, where the inner planet's orbit decays at a rate that is faster than that expected from the direct tidal effect, while the outer planet gains orbital energy. In Section 4, we discuss the extension of our formalism to multi-resonant systems. Subsequently, we conclude and discuss our results in Section 5.

\section{CONSERVATIVE DYNAMICS OF A RESONANT PLANETARY PAIR}

Resonant dynamics of planetary pairs have been studied by numerous authors in the past (see Chapter 8 of Murray \& Dermott 1999 and references therein). This work builds on their contributions.

Our eventual goal is to construct an analytical model for the long-term evolution of resonant orbits under dissipative effects. Before complicating the picture with dissipation, however, we must first build a purely analytical model for conservative resonant interactions. Thus, in this section, we shall derive a simple, physically intuitive closed-form solution for the time evolution of a resonant planetary pair. Accordingly, we shall first work in the spirit of classical perturbation theory (e.g., Message 1966; Peale 1986) and employ numerical calculations primarily as a means of confirmation.

Let us begin by considering a quasi-integrable Hamiltonian of the form

$$
\mathcal{H}=\mathcal{H}_{\text {kep }}+\mathcal{H}_{\text {res }}+\mathcal{O}\left(e^{2}, i^{2}\right),
$$

where

$$
\mathcal{H}_{\text {kep }}=-G \frac{M m_{1}}{2 a_{1}}-G \frac{M m_{2}}{2 a_{2}}
$$

is the Keplerian Hamiltonian and

$$
\begin{aligned}
\mathcal{H}_{\text {res }}= & -G \frac{m_{1} m_{2}}{a_{2}}\left(f_{\text {res }}^{(1)} e_{1} \cos \left(k \lambda_{2}-(k-1) \lambda_{1}-\varpi_{1}\right)\right. \\
& \left.+f_{\text {res }}^{(2)} e_{2} \cos \left(k \lambda_{2}-(k-1) \lambda_{1}-\varpi_{2}\right)\right)
\end{aligned}
$$

is the first-order $k: k-1, k \in \mathbb{Z}$ resonant perturbation. Here, the orbital elements take on their standard notation: $M$ is the mass of the central star and $m_{1}$ and $m_{2}$ are the masses of the planets with the subscript 1 and 2 referring to the inner and outer planets, respectively. The quantities $f_{\text {res }}^{(1)}$ and $f_{\text {res }}^{(2)}$ depend on the semimajor axis ratio $\left(a_{1} / a_{2}\right)$ only and are tabulated in the literature (see, for example, Murray \& Dermott 1999).

Because Keplerian orbital elements are not canonically conjugated, we revert to Poincaré variables for further calculations:

$$
\begin{gathered}
\Lambda=m \sqrt{G M a}, \quad \lambda=\mathcal{N}+\varpi \\
\Gamma=\Lambda\left(1-\sqrt{1-e^{2}}\right) \approx \Lambda e^{2} / 2, \quad \gamma=-\varpi,
\end{gathered}
$$

where $\mathcal{N}$ is the mean anomaly and the indexes 1,2 are omitted for simplicity. In terms of the Poincare variables, the Hamiltonians, $\mathcal{H}_{\text {kep }}$ and $\mathcal{H}_{\text {res }}$, read:

$$
\begin{gathered}
\mathcal{H}_{\text {kep }}=-\frac{G^{2} M^{2} m_{1}^{3}}{2 \Lambda_{1}^{2}}-\frac{G^{2} M^{2} m_{2}^{3}}{2 \Lambda_{2}^{2}} \\
\mathcal{H}_{\text {res }}=-\frac{G^{2} M m_{1} m_{2}^{3}}{\Lambda_{2}^{2}}\left(f_{\text {res }}^{(1)} \sqrt{\frac{2 \Gamma_{1}}{\Lambda_{1}}} \cos \left(k \lambda_{2}-(k-1) \lambda_{1}+\gamma_{1}\right)\right. \\
\left.+f_{\text {res }}^{(2)} \sqrt{\frac{2 \Gamma_{2}}{\Lambda_{2}}} \cos \left(k \lambda_{2}-(k-1) \lambda_{1}+\gamma_{2}\right)\right)
\end{gathered}
$$

As already implied by Equation (1), we shall work to first order in eccentricity, neglecting secular effects and resonances of the order of greater than unity. Generally, $\mathcal{H}$ only constitutes a good approximation to the true dynamics of a planetary pair in the vicinity of a mean-motion resonance.

Because the perturbation $\mathcal{H}_{\text {res }}$ is of the order of $e$, we expect that the semimajor axes can change by $\mathcal{O}(\sqrt{e})$ relative to their nominal, resonant values. Thus, we expand the terms in $\mathcal{H}_{\text {kep }}$ to second order in $\delta \Lambda=\Lambda-[\Lambda]$, where $[\Lambda]$ is the nominal value of $\Lambda$ :

$$
\begin{aligned}
\mathcal{H}_{\mathrm{kep}}= & -\frac{G^{2} M^{2} m_{1}^{3}}{2[\Lambda]_{1}^{2}}+\frac{G^{2} M^{2} m_{1}^{3}}{[\Lambda]_{1}^{3}} \delta \Lambda_{1}-\frac{3 G^{2} M^{2} m_{1}^{3}}{2[\Lambda]_{1}^{4}} \delta \Lambda_{1}^{2} \\
& -\frac{G^{2} M^{2} m_{2}^{3}}{2[\Lambda]_{2}^{2}}+\frac{G^{2} M^{2} m_{2}^{3}}{[\Lambda]_{2}^{3}} \delta \Lambda_{2}-\frac{3 G^{2} M^{2} m_{2}^{3}}{2[\Lambda]_{2}^{4}} \delta \Lambda_{2}^{2} \\
& +\mathcal{O}\left(\delta \Lambda_{1}^{3}, \delta \Lambda_{2}^{3}\right) .
\end{aligned}
$$

Consistently, we evaluate $\mathcal{H}_{\text {res }}$ in Equation (6) at $[\Lambda]$, as it is already of the order of $\mathcal{O}(e)$. Constant terms are dynamically unimportant and can thus be dropped from the Hamiltonian, implying $\delta \Lambda \rightarrow \Lambda$ and $\delta \Lambda^{2} \rightarrow \Lambda^{2}-2 \Lambda[\Lambda]$ :

$$
\begin{aligned}
\mathcal{H}_{\text {kep }}= & \frac{4 G^{2} M^{2} m_{1}^{3} \Lambda_{1}}{\left[\Lambda_{1}\right]^{3}}+\frac{4 G^{2} M^{2} m_{2}^{3} \Lambda_{2}}{\left[\Lambda_{2}\right]^{3}} \\
& -\frac{3 G^{2} M^{2} m_{1}^{3} \Lambda_{1}^{2}}{2\left[\Lambda_{1}\right]^{4}}-\frac{3 G^{2} M^{2} m_{2}^{3} \Lambda_{2}^{2}}{2\left[\Lambda_{2}\right]^{4}} .
\end{aligned}
$$

Note that the planetary mean motion is given by

$$
n=\frac{d \lambda}{d t}=\frac{\partial \mathcal{H}_{\mathrm{kep}}}{\partial \Lambda}=\frac{G^{2} M^{2} m^{3}}{\Lambda^{3}} .
$$



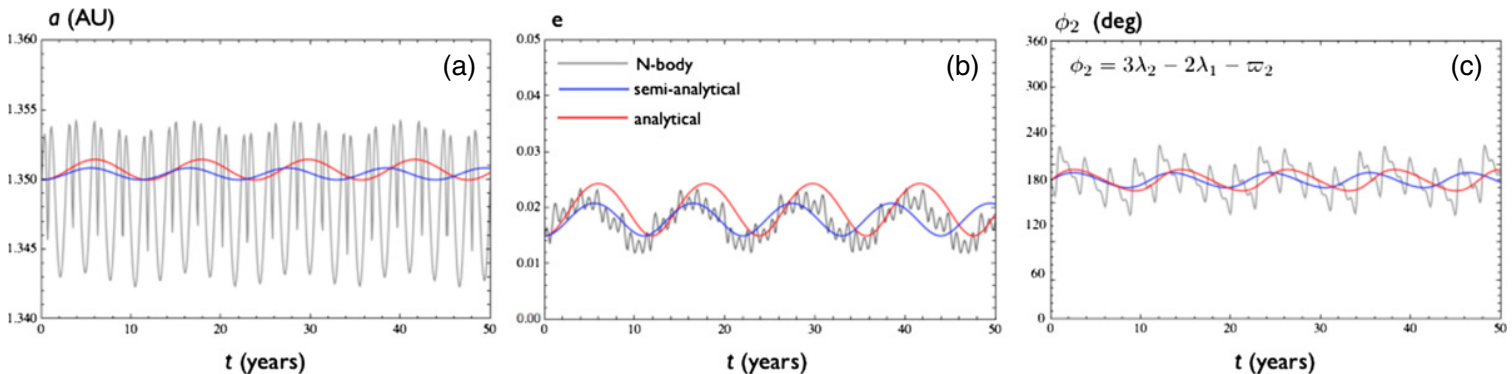

Figure 2. Orbital evolution of a nearly mass-less $\left(m=10^{-10} M_{\odot}\right)$ particle in an interior 2:1 mean-motion resonance with a Jupiter-mass object $\left(m=10^{-3} M_{\odot}\right)$ with a semimajor axis of $a=1 \mathrm{AU}$. The evolution is shown over 50 orbital periods of the perturbing object, corresponding to $\sim 5$ resonant cycles. The panels (A)-(C) show the variation in the particle's semimajor axes, eccentricity, and the critical resonant angle, respectively. The red curve was obtained analytically utilizing the framework developed in Section 2. The blue curve was obtained by numerically integrating the equations of motion that arise from the Hamiltonians (6) and (7). The gray curve is a result of a direct $N$-body simulation.

(A color version of this figure is available in the online journal.)

As a result, $\mathcal{H}_{\text {kep }}$ can be rewritten in a compact form

$$
\mathcal{H}_{\text {kep }}=4\left([n]_{1} \Lambda_{1}+[n]_{2} \Lambda_{2}\right)-\frac{3}{2}\left([h]_{1} \Lambda_{1}^{2}+[h]_{2} \Lambda_{2}^{2}\right),
$$

where $[h]=[n] /[\Lambda]=1 /\left(m /[a]^{2}\right)$.

Although $\mathcal{H}_{\text {kep }}$ is now expressed in a simple form, $H_{\text {res }}$ remains cumbersome largely due to the formulation of the resonant angles which appear as cosine arguments. Let us employ a canonical transformation of coordinates, utilizing the following generating function of the second kind:

$$
\begin{aligned}
F_{2}= & \lambda_{1} \Psi_{1}+\lambda_{2} \Psi_{2}+\left(k \lambda_{2}-(k-1) \lambda_{1}+\gamma_{1}\right) \Phi_{1} \\
& +\left(k \lambda_{2}-(k-1) \lambda_{1}+\gamma_{2}\right) \Phi_{2},
\end{aligned}
$$

where $\Psi$ and $\Phi$ are new momenta. Upon application of the transformation equations

$$
\Lambda=\frac{\partial F}{\partial \lambda} \quad \Gamma=\frac{\partial F}{\partial \gamma}
$$

we obtain new canonically conjugated action-angle variables

$$
\begin{array}{ll}
\Psi_{1}=\Lambda_{1}+(k-1)\left(\Phi_{1}+\Phi_{2}\right) & \psi_{1}=\lambda_{1} \\
\Psi_{2}=\Lambda_{2}-k\left(\Phi_{1}+\Phi_{2}\right) & \psi_{2}=\lambda_{2} \\
\Phi_{1}=\Gamma_{1} & \phi_{1}=k \lambda_{2}-(k-1) \lambda_{1}+\gamma_{1} \\
\Phi_{2}=\Gamma_{2} & \phi_{2}=k \lambda_{2}-(k-1) \lambda_{1}+\gamma_{2} .
\end{array}
$$

In terms of these variables, the resonant contribution to $\mathcal{H}$ is expressed as follows:

$$
\mathcal{H}_{\text {res }}=-\frac{G^{2} M m_{1} m_{2}^{3}}{[\Lambda]_{2}^{2}}\left(f_{\text {res }}^{(1)} \sqrt{\frac{2 \Phi_{1}}{[\Lambda]_{1}}} \cos \left(\phi_{1}\right)+f_{\text {res }}^{2} \sqrt{\frac{2 \Phi_{2}}{[\Lambda]_{2}}} \cos \left(\phi_{2}\right)\right),
$$

while the Keplerian contribution reads

$$
\begin{aligned}
\mathcal{H}_{\text {kep }}= & 4[n]_{1}\left(\Psi_{1}-(k-1)\left(\Phi_{1}+\Phi_{2}\right)\right) \\
& +4[n]_{2}\left(\Psi_{2}+k\left(\Phi_{1}+\Phi_{2}\right)\right) \\
& -\frac{3}{2}[h]_{1}\left(\Psi_{1}-(k-1)\left(\Phi_{1}+\Phi_{2}\right)\right)^{2} \\
& -\frac{3}{2}[h]_{2}\left(\Psi_{2}+k\left(\Phi_{1}+\Phi_{2}\right)^{2}\right) .
\end{aligned}
$$

The transformation to new variables allows us to make further simplifications to $\mathcal{H}_{\text {kep }}$. Specifically, because $\partial H / \partial \psi=0$,
$\Psi_{1}$ and $\Psi_{2}$ are constants of motion, allowing us to drop additional terms. It is further instructive to recall that $\Phi \propto e^{2}$. Consequently, if $e \ll 1$, then nonlinear terms proportional to $\Phi_{1}^{2}$, $\Phi_{2}^{2}$, and $\Phi_{1} \Phi_{2}$ can be neglected. This approximation filters out chaotic dynamics from the Hamiltonian and therefore will not yield an adequate representation of the evolution of the system in the resonances overlap region (Chirikov 1979; Wisdom 1980). However, as will be shown below, this assumption is well satisfied in the calculations of interest. Upon making these simplifications, the Keplerian Hamiltonian is simply

$$
\begin{aligned}
\mathcal{H}_{\text {kep }}= & \left(4\left(k[n]_{2}-(k-1)[n]_{1}\right)\right) \\
& \left.+3\left([h]_{1}(k-1) \Psi_{1}-[h]_{2} k \Psi_{2}\right)\right)\left(\Phi_{1}+\Phi_{2}\right) .
\end{aligned}
$$

Note that by definition, $\left(k[n]_{2}-(k-1)[n]_{1}\right)=0$ because it signifies exact resonance. As a result, only terms proportional to $[h]$ remain in $\mathcal{H}_{\text {kep }}$.

The full Hamiltonian now takes on a very simple form

$$
\mathcal{H}=\eta\left(\Phi_{1}+\Phi_{2}\right)+\alpha \sqrt{2 \Phi_{1}} \cos \left(\phi_{1}\right)+\beta \sqrt{2 \Phi_{2}} \cos \left(\phi_{2}\right),
$$

where

$$
\eta=3\left([h]_{1}(k-1) \Psi_{1}-[h]_{2} k \Psi_{2}\right)
$$

is related to the circulation frequency of the critical angles in an unperturbed case $\left(m_{1}=m_{2}=0\right)$ and is thus a measure of proximity of the planetary pair to exact Keplerian resonance (note that $\eta \rightarrow 0$ as $\Lambda \rightarrow[\Lambda]$ and $\Phi \rightarrow 0$, corresponding to $\Psi=[\Lambda])$ while

$$
\begin{aligned}
& \alpha=-\frac{G^{2} M m_{1} m_{2}^{3}}{[\Lambda]_{2}^{2}} \frac{f_{\text {res }}^{(1)}}{\sqrt{[\Lambda]_{1}}} \\
& \beta=-\frac{G^{2} M m_{1} m_{2}^{3}}{[\Lambda]_{2}^{2}} \frac{f_{\text {res }}^{(2)}}{\sqrt{[\Lambda]_{2}}}
\end{aligned}
$$

are the strengths of the resonances. It is noteworthy that the Hamiltonian (18) represents two decoupled Hamiltonians, each of which has a form similar to the "second fundamental model of resonance" (Henrard \& Lamaitre 1983), apart from the missing term, proportional to $\Phi^{2}$, that we have neglected.

In the coordinates used up to now, the equations of motion are singular at $\Phi=0$. However, this singularity can be overcome by switching to mixed Cartesian coordinates

$$
x=\sqrt{2 \Phi} \sin (\phi) \quad y=\sqrt{2 \Phi} \cos (\phi)
$$



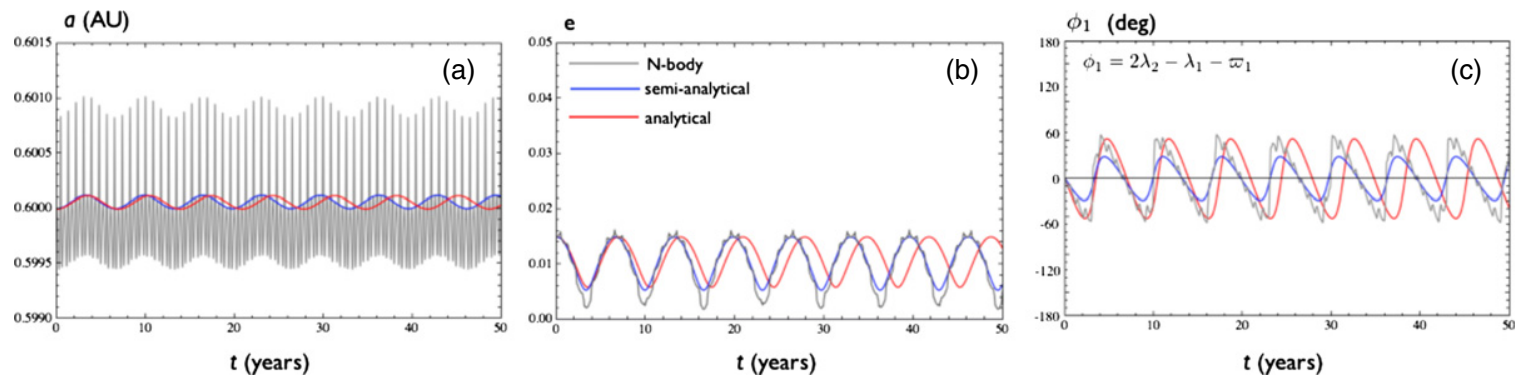

Figure 3. Orbital evolution of a nearly mass-less $\left(m=10^{-10} M_{\odot}\right)$ particle in an exterior 3:2 mean-motion resonance with a Jupiter-mass object $\left(m=10^{-3} M_{\odot}\right)$ with a semimajor axis of $a=1 \mathrm{AU}$. The evolution is shown over 50 orbital periods of the perturbing object, corresponding to $\sim 7$ resonant cycles. As in Figure 2 , the panels (A)-(C) show the variation in the particle's semimajor axes, eccentricity, and the critical resonant angle, respectively. The red curve was obtained analytically utilizing the framework developed in Section 2. The blue curve was obtained by numerically integrating the equations of motion that arise from the Hamiltonians (6) and (7). The gray curve is a result of a direct $N$-body simulation.

(A color version of this figure is available in the online journal.)

via a contact transformation (here, $x$ is identified as the coordinate and $y$ as the momentum). In these coordinates, the Hamiltonian reads

$$
\mathcal{H}=\frac{\eta}{2}\left(x_{1}^{2}+y_{1}^{2}+x_{2}^{2}+y_{2}^{2}\right)+\alpha y_{1}+\beta y_{2}
$$

Accordingly, the equations of motion are

$$
\begin{aligned}
\frac{d x_{1}}{d t} & =\frac{\partial \mathcal{H}}{\partial y_{1}}=\alpha+\eta y_{1} \\
\frac{d x_{2}}{d t} & =\frac{\partial \mathcal{H}}{\partial y_{2}}=\beta+\eta y_{2} \\
\frac{d y_{1}}{d t} & =-\frac{\partial \mathcal{H}}{\partial x_{1}}=-\eta x_{1} \\
\frac{d y_{2}}{d t} & =-\frac{\partial \mathcal{H}}{\partial x_{2}}=-\eta x_{2} .
\end{aligned}
$$

Although we can continue to work in terms of the mixed Cartesian coordinates, the equations of motion can be re-written in a more compact form by treating $x$ and $y$ as imaginary and real components of a single complex variable

$$
z=\imath x+y \text {. }
$$

Now, the equations of motion can be written down concisely

$$
\begin{aligned}
& \frac{d z_{1}}{d t}=\imath \alpha+\imath \eta z_{1} \\
& \frac{d z_{2}}{d t}=\imath \beta+\imath \eta z_{1},
\end{aligned}
$$

and admit the analytical solutions

$$
\begin{aligned}
& z_{1}=-\frac{\alpha}{\eta}+\mathcal{C}_{1} \exp (\imath \eta t) \\
& z_{2}=-\frac{\beta}{\eta}+\mathcal{C}_{2} \exp (\imath \eta t)
\end{aligned}
$$

where $\mathcal{C}_{1}$ and $\mathcal{C}_{2}$ are (possibly complex) constants of integration. Note that except for a dependence of the leading term on $z$, Equations (25) are analogous to the complex formulation of the Laplace-Lagrange theory for secular interactions $(\mathrm{Wu}$ \& Goldreich 2002; Batygin \& Laughlin 2011), although the variables take on a different meaning.

Within the context of this model, variations in semimajor axes can be derived from the fact that $\Psi$ remain constants of motion. Examples of the application of the theory are presented in Figures 2 and 3. In both of the illustrated cases, a nearly mass-less $\left(m=10^{-10} M_{\odot}\right)$ particle is perturbed by a Jupiter-mass object $\left(m=10^{-3} M_{\odot}\right)$ with a semimajor axis of $a=1 \mathrm{AU}$. Figure 2 shows an interior 2:1 meanmotion resonance while Figure 3 shows an exterior 3:2 meanmotion resonance. The red curves denote analytical theory, the blue curves represent a numerical integration of the nonlinear perturbative Hamiltonians (6) and (7), and the gray curves are the results of numerical $N$-body simulations, performed using the hybrid algorithm of the orbital integration software package mercury6 (Chambers 1999). Note that as a consequence of the simplifications made in order to express the analytical solution in closed form, the blue (nonlinear perturbative) curve has a slightly different frequency and amplitude of oscillation relative to the red (analytical) curve, although the two curves exhibit the same qualitative behavior. However, in addition to the resonant variations, the gray ( $N$-body) curve shows non-resonant, short-period oscillations, that are filtered out by retaining only the resonant terms in the Hamiltonian. These short-periodic oscillations are unimportant to the problem at hand, as they do not contribute to the time averages of the resonant angles. Note also that, although the particles in both examples are relatively far away from nominal resonance, the critical angles remain in libration.

\section{DISSIPATIVE DYNAMICS OF A RESONANT PLANETARY PAIR}

There exists an abundance of circumstances where the evolution of a planetary system cannot be described in terms of strictly conservative interactions. For example, planets embedded in protoplanetary disks experience dissipative forces exerted by the gaseous nebula (Lee \& Peale 2002), while planets that reside on orbits that are in close proximity to their host stars are subject to tidal friction (Bodenheimer et al. 2001; in this work, we shall concentrate on the latter). In the extrasolar context, tidal dissipation usually results in the decay of orbital eccentricity and semimajor axes.

With the exception of special configurations, the characteristic timescales for the decay of eccentricity and semimajor axes differ significantly (often by orders of magnitude). This is in part because the changes in eccentricity are controlled by the rate of angular momentum exchange in the system, while changes in the semimajor axes are largely governed by the rate of energy dissipation, which is usually a much slower process. As a result, for the purposes of this work, we shall invoke separation 
of timescales and treat the decays of $e$ and $a$ independently. For $e \ll 1$, the orbit-averaged rate of tidal eccentricity decay is given by (Goldreich \& Soter 1966)

$$
\left(\frac{d e}{d t}\right)_{\text {tide }}=-e \frac{21[n]}{2} \frac{k}{Q} \frac{M}{m}\left(\frac{R}{[a]}\right)^{5}=-\frac{e}{\tau_{e}},
$$

where $k$ is the planetary Love number, $Q$ is the tidal quality factor (note that dissipation within the host star is neglected as usual), and $R$ is the planetary radius. Noting that $|z| \simeq e \sqrt{[\Lambda]}$, it is trivial to incorporate eccentricity decay into Equations (25):

$$
\begin{aligned}
& \frac{d z_{1}}{d t}=\imath \alpha+\imath \eta z_{1}-\frac{z_{1}}{\tau_{e_{1}}} \\
& \frac{d z_{2}}{d t}=\imath \beta+\imath \eta z_{1}-\frac{z_{2}}{\tau_{e_{2}}} .
\end{aligned}
$$

Since the equations of motion remain linear in $z$, they admit solutions that are formally similar to (26):

$$
\begin{aligned}
& z_{1}=-\frac{\alpha}{\eta+\imath / \tau_{e_{1}}}+\mathcal{C}_{1} \exp \left(\imath \eta t-t / \tau_{e_{1}}\right) \\
& z_{2}=-\frac{\beta}{\eta+\imath / \tau_{e_{2}}}+\mathcal{C}_{2} \exp \left(\imath \eta t-t / \tau_{e_{2}}\right) .
\end{aligned}
$$

Note that the eccentricity damping timescale of the second body in the equation above is $\tau_{e_{2}}$. Depending on $Q$, this timescale can appear to greatly exceed $\tau_{e_{1}}$. However, it is important to keep in mind that in reality, variations in $\phi_{1}$ and $\phi_{2}$ are coupled because both give rise to changes in the planetary semimajor axes. This means that tidal dissipation of the inner planet's eccentricity also damps the outer planet's eccentricity resonantly. Furthermore, the first and the second planet are also coupled through a secular term of the form $\mathcal{H}_{\sec } \propto e_{1} e_{2} \cos \left(\varpi_{1}-\varpi_{2}\right)$ that we have neglected in the Hamiltonian. Through this secular interaction, tidal damping on $e_{1}$ is translated to $e_{2}$ as well (albeit on a longer timescale), even if there is no direct damping on $e_{2}$ (i.e., $\tau_{e_{2}}=\infty$; Wu \& Goldreich 2002; Mardling 2007).

Because the dissipation is applied directly on the actions, Hamiltonian properties of the solution such as the conservation of phase-space area bounded by the orbit are destroyed. On a timescale of approximately a few $\tau_{e}$, the second terms in the solutions (29) will decay away, making the phase-space area bounded by the orbit tend to zero. This has a number of important physical implications. First of all, this removes the dependence of the long-term $\left(t \gg \tau_{z}\right)$ solution on the initial conditions. Second, the fact that explicit time dependence of the solution is also lost, suggests that the eccentricity dynamics falls onto a fixed point attractor, characterized by constant actions (i.e., eccentricities) and angles (Batygin \& Morbidelli 2011). Specifically, assuming that $1 / \tau_{e} \ll(|\alpha / \eta|,|\beta / \eta|)$, we obtain

$$
\begin{aligned}
& e_{1} \rightarrow-\sqrt{\frac{1}{[\Lambda]_{1}} \frac{\alpha}{\eta}} \quad \phi_{1} \rightarrow \frac{1}{\eta \tau_{e_{1}}} \\
& e_{2} \rightarrow+\sqrt{\frac{1}{[\Lambda]_{2}} \frac{\beta}{\eta}} \quad \phi_{2} \rightarrow \pi-\frac{1}{\eta \tau_{e_{2}}},
\end{aligned}
$$

where the involved quantities are given in terms of Keplerian orbital elements by Equations (4), (19), and (20). Mathematically, $\Delta \phi \approx \pi$ arises from the fact that for all first-order resonances, $f_{\text {res }}^{(1)}<0$, while $f_{\text {res }}^{(2)}>0$. A physical consequence of this fact is that all stationary resonant planetary pairs will be apsidally anti-aligned.

The above solution diverges as $\eta \rightarrow 0$ and gives positive values of $e_{1}, e_{2}$ only if $\eta<0$. This is because the stable equilibrium points of the resonance are always characterized by period ratios $n_{1} / n_{2}$ that are larger than the exact resonant value. This is a well-known fact for first-order resonances (see, for example, Chapter 9 of Morbidelli 2002). ${ }^{4}$

The solution (29) also illustrates that, beyond the transient equilibration period, the eccentricity ratio remains constant for all time, since $\alpha$ and $\beta$ are strictly constant, while the actual eccentricity values depend only on $\eta$, i.e., on the proximity of the planets to exact resonance. ${ }^{5}$ Because we have restricted ourselves to only a linear treatment of eccentricity, this solution fails close to exact resonance, where equilibrium eccentricities can be quite large. However, this limitation only proves problematic in a rather narrow region of parameter space.

Thus far, we have only considered the relatively fast equilibration of orbital eccentricities and critical angles. Let us now turn our attention to the truly long-term evolution of the system and the associated change in the semimajor axes. There are two effects of importance. The simpler of the two effects is direct tidal damping of semimajor axes. To leading order in $e$ (Goldreich \& Soter 1966),

$$
\left(\frac{d a}{d t}\right)_{\text {tide }}=-2 e^{2} \frac{a}{\tau_{e}} .
$$

Recall that the eccentricities converge onto quasi-fixed points. Thus, in terms of Poincare variables, the tidal decay of semimajor axes can be written as

$$
\begin{aligned}
& \left(\frac{d \Lambda_{1}}{d t}\right)_{\text {tide }}=-2 \frac{\Gamma_{1}}{\tau_{e_{1}}} \simeq-\frac{1}{\tau_{e_{1}}} \frac{\alpha^{2}}{\eta^{2}} \\
& \left(\frac{d \Lambda_{2}}{d t}\right)_{\text {tide }}=-2 \frac{\Gamma_{2}}{\tau_{e_{2}}} \simeq-\frac{1}{\tau_{e_{2}}} \frac{\beta^{2}}{\eta^{2}} .
\end{aligned}
$$

For similar physical planetary parameters (including quality factors) and eccentricities, tidal evolution will cause orbits to diverge, since $\tau_{e_{2}} / \tau_{e_{1}} \sim(k / k-1)^{10 / 3}$, although both semimajor axes drift in the same direction (i.e., decay toward the central star).

The second, more subtle effect is the resonant divergence of the orbits, forced by eccentricity damping. As shown above, tidal decay of eccentricity causes the critical angles to collapse onto stable fixed points. However, these fixed points are slightly offset from the actual foci. This offset results in a monotonic drift of the semimajor axes in opposite directions. To understand this, let us return to our original formulation of the Hamiltonian. An application of Hamilton's equations to Hamiltonian (7), evaluated on $e$ and $\phi$ given in Equation (29), yields

$$
\begin{aligned}
& \left(\frac{d \Lambda_{1}}{d t}\right)_{\mathrm{res}}=(1-k)\left(\frac{1}{\tau_{e_{1}}} \frac{\alpha^{2}}{\eta^{2}}+\frac{1}{\tau_{e_{2}}} \frac{\beta^{2}}{\eta^{2}}\right) \\
& \left(\frac{d \Lambda_{2}}{d t}\right)_{\mathrm{res}}=k\left(\frac{1}{\tau_{e_{1}}} \frac{\alpha^{2}}{\eta^{2}}+\frac{1}{\tau_{e_{2}}} \frac{\beta^{2}}{\eta^{2}}\right),
\end{aligned}
$$

where we have made the small angle approximation: $\sin (\phi) \simeq \phi$. Note that the rate of change of the outer semimajor axis is

\footnotetext{
4 This is true only for small to moderate eccentricity values.

5 Note that at the level of approximation which we have employed, the eccentric contribution to $\Psi$ can be neglected, since $\Phi \propto e^{2}$. Thus, in the definition of $\eta$ in Equation (18), it can be safely assumed that $\Psi \simeq \Lambda$.
} 

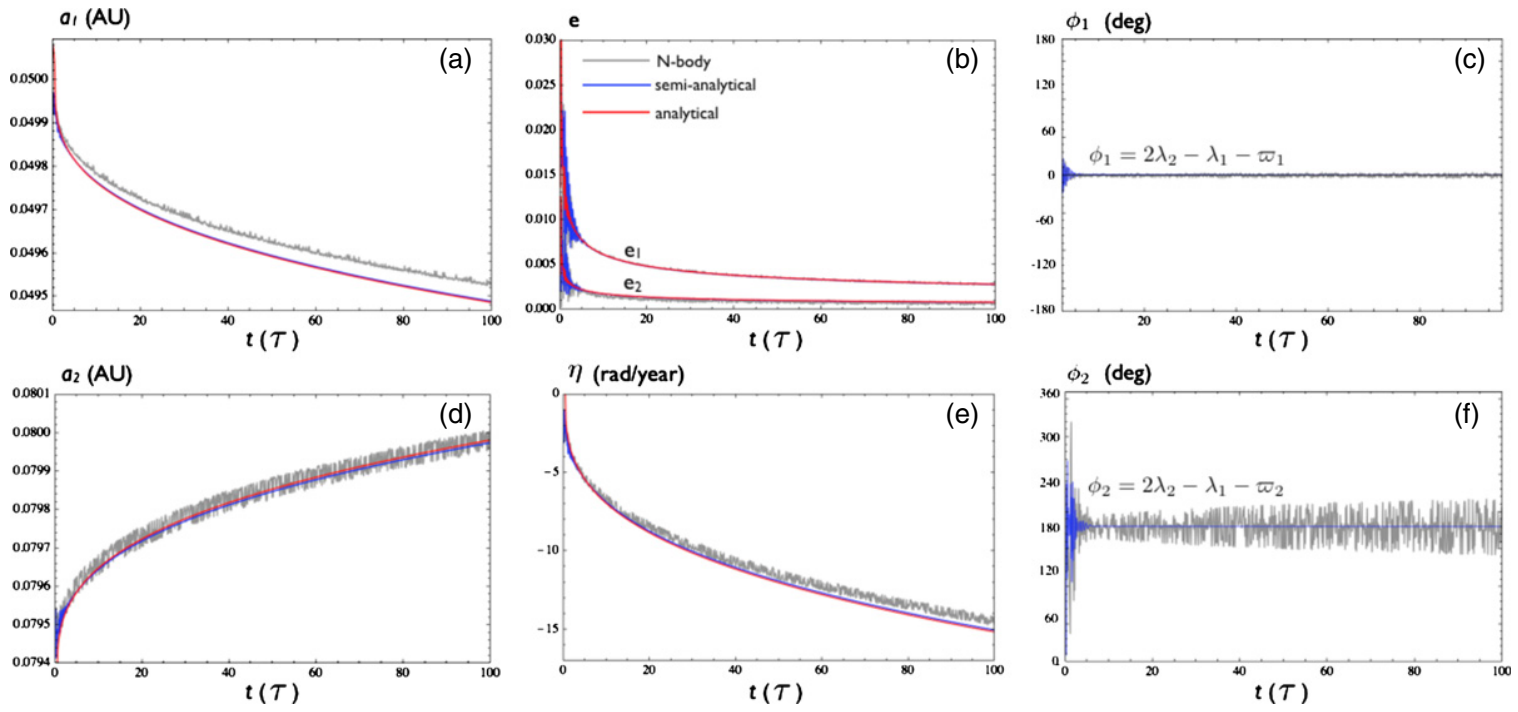

Figure 4. Dissipative evolution of an equal-mass $\left(m_{1}=m_{2}=10^{-4} M\right)$ planetary pair in a 2:1 mean-motion resonance over $t / \tau=100$ circularization timescales. Panels (A) and (D) show the evolution of the planetary semimajor axes. Note that at all times, dissipative interactions give rise to a monotonic divergence of the orbits. This can be further inferred from panel (E) which shows the measure of proximity to exact resonance, $\eta<0$ monotonically decreasing. Panels (C) and (F) show the evolution of the critical angles. Note that the system attains a state of quasi-equilibrium after $t \sim 5 \tau$. Accordingly, the eccentricity evolution also becomes quasi-stationary after the critical angles collapse to a near-focal state. The red curves were obtained analytically utilizing the framework developed in Section 3 . The blue curves were obtained by numerically integrating the equations of motion that arise from the Hamiltonians (6) and (7), augmented with a simple parameterization of tidal dissipation (i.e., Equations (27) and (31)). The gray curves were computed numerically with a direct $N$-body simulation where dissipation has been taken into account using the tidal framework of Eggleton et al. (1998).

(A color version of this figure is available in the online journal.)

positive definite, while that of the inner semimajor axis is negative definite. In other words, eccentricity damping always results in the drift of the semimajor axes in opposite directions, as anticipated above.

The long-term behavior of the resonance can be understood by combining Equations (32), (18), and (29) to yield an equation of motion ${ }^{6}$ for $\eta$ :

$$
\frac{d \eta}{d t}=-\frac{3\left([h]_{1}(k-1)+[h]_{2} k\right)\left(k \alpha^{2} \tau_{e_{2}}+(k-1) \beta^{2} \tau_{e_{1}}\right)}{\eta^{2} \tau_{e_{1}} \tau_{e_{2}}} .
$$

This equation admits the solution

$$
\begin{aligned}
\eta= & (-1)^{2 / 3}\left\{\eta_{0}^{3}-\frac{9 t}{\tau_{e_{1}} \tau_{e_{2}}}\left(k[h]_{2}+(k-1)[h]_{1}\right)\right. \\
& \left.\times\left(k \alpha^{2} \tau_{e_{2}}+(k-1) \beta^{2} \tau_{e_{1}}\right)\right\}^{1 / 3}
\end{aligned}
$$

where $\eta_{0}<0$ is an initial condition, corresponding to the initial value of $\eta$ for a resonant equilibrium (which needs to be negative as shown in Equation (29)). Note that the solution (34) monotonically decreases in time, leading to an increase in the absolute value of $\eta$, i.e., an increase in the distance between the semimajor axes of the planets relative to the Keplerian location of the resonance. The same $\eta \propto t^{1 / 3}$ dependence was observed in the simulations of Papaloizou \& Terquem (2010). Meanwhile, the resonant angles, $\phi$ will maintain a near-null libration width leading to quasi-constant eccentricity evolution.

\footnotetext{
6 Here, the direct tidal and resonant contributions to the evolution of the semimajor axes have been combined assuming that there are no indirect terms in the disturbing function i.e., the $\beta$ 's in Equations (32) and (33) are identical. This is true for all first-order resonant arguments, except $\phi=2 \lambda_{2}-\lambda_{1}-\varpi_{2}$. In the exceptional case, the indirect terms must be properly taken into account (this is done in the calculation shown in Figure 4).
}

Figure 4 presents an example of such evolution. In the case shown, two equal-mass $\left(m_{1}=m_{2}=10^{-4} M\right)$ planets start out in exact $2: 1$ resonance with $a_{1}=0.05 \mathrm{AU}$, $e_{1}=e_{2}=0.01$, and randomly chosen angles. In this calculation, we have set $\tau_{e_{1}}=\tau_{e_{2}}$ and use this dissipation timescale as a unit of time (this is validated as a result of the adiabatic nature of the evolution). As above, each panel shows three separate calculations. Blue curves represent solutions obtained by numerically integrating the nonlinear Hamiltonians (7) and (6) in the presence of tidal dissipation (parameterized by Equations (27) and (31)), red curves stem from the fully analytical framework presented in this section, while the gray curves result from an $N$-body simulation, where tidal and general relativistic interactions are accounted for directly (Mardling \& Lin 2002) and integrated using the Bulirsch-Stoer algorithm (Press et al. 1992). As predicted by the theoretical arguments above, after a few $(\sim 5)$ circularization timescales, the system collapses onto a fixed state where the critical angles approach their respective foci and the variations in eccentricities damp out. Once a quasi-stationary configuration is achieved, the orbits slowly diverge while the two resonant angles $\phi_{1}$ and $\phi_{2}$ remain in libration, which means, strictly speaking, that the resonant configuration is maintained (although the separatrix associated with the resonance disappears at a certain $\eta$-see Delisle et al. 2012; Peale 1986).

Importantly, when dissipation is applied to a resonant pair, the outer orbit drifts outward, gaining orbital energy. This behavior is in contrast with a naive application of standard tidal theory to the individual planets, where both planets are taken to drift inward, and facilitates a faster divergence of the orbits.

As already mentioned above, the long-term evolution of the system is adiabatic: the characteristic timescale for significant orbital divergence greatly exceeds the resonant interaction timescale. Conveniently, this fact renders orbital divergence to be a scale-free process. In other words, the fractional 


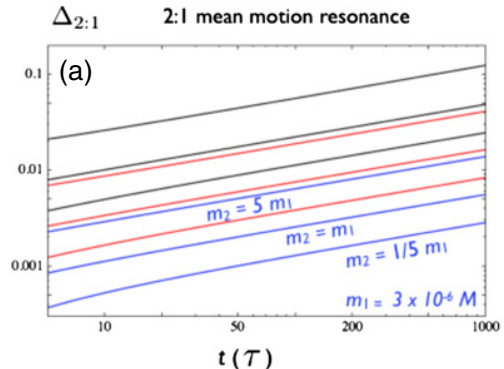

$t(\tau)$

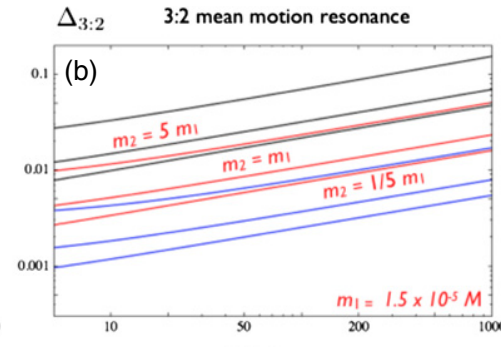

$t(\tau)$

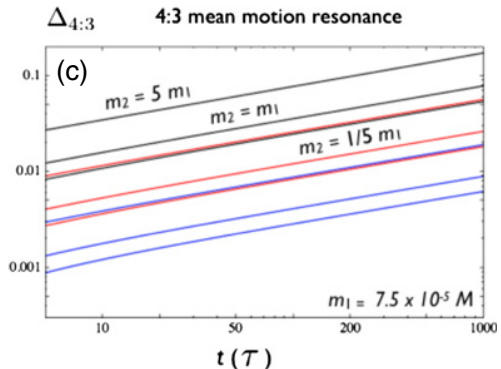

$t(\tau)$

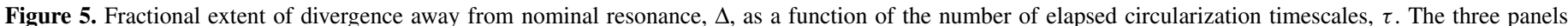

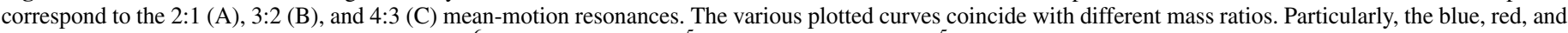

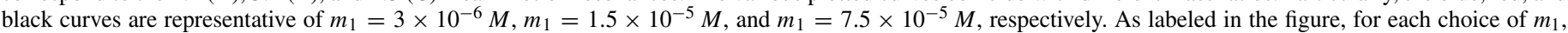

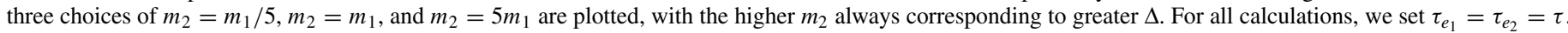

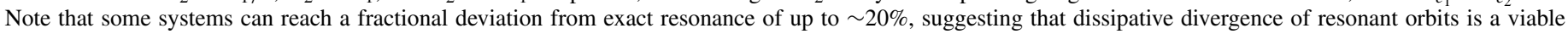
mechanism for production of planet pairs that reside significantly outside of nominal resonance.

(A color version of this figure is available in the online journal.)

divergence away from exact resonance is not explicitly controlled by the actual semimajor axes or masses of the planets, but rather by the mass ratios $\left(m_{1} / m_{2}, m / M\right)$ and the number of elapsed circularization timescales, $t / \tau$. Taking advantage of this, we have delineated the fractional extent of orbital divergence,

$$
\Delta_{\mathrm{k}: \mathrm{k}-1}=\frac{n_{1} / n_{2}-k /(k-1)}{k / k-1}
$$

as a function of elapsed dimensionless time, $t / \tau$, for an array for planetary mass ratios. These results are demonstrated in Figure 5, where the three panels correspond to the 2:1 (A), 3:2 (B), and 4:3 (C) mean-motion resonances. In the figure, blue curves correspond to $m_{1}=3 \times 10^{-6} \mathrm{M}$, red curves to $m_{1}=1.5 \times 10^{-5} \mathrm{M}$, and black curves to $m_{1}=7.5 \times 10^{-5} \mathrm{M}$. For each color-coded choice of $m_{1}$, three choices of $m_{2}=m_{1} / 5$, $m_{2}=m_{1}$, and $m_{2}=5 m_{1}$ are plotted, with the higher $m_{2}$ always corresponding to greater $\Delta$. Note that after $t / \tau \gtrsim 100$, the more massive examples presented in Figure 5 can reside more than $\sim 10 \%$ away from nominal resonance. This points to the viability of creating the near-resonant overpopulation observed in the Kepler sample by the mechanism discussed here.

\section{DISSIPATIVE DYNAMICS OF MULTI-RESONANT PLANETARY SYSTEMS}

There is considerable motivation to extend the above analysis to systems made of more than two planets, where each body is in resonance with all of its neighbors, as such systems appear to be common in nature. Perhaps the best-studied example of a multiresonant system is the Galilean satellites, where both satellite pairs are locked in 2:1 mean-motion resonances, leading to the libration of the Laplace argument. In the collection of confirmed extrasolar planets, examples of multi-resonant systems include the GL876 system - where the Laplace resonance is directly observed (Rivera et al. 2010) — the HD40307 (Mayor et al. 2009) system - which contains three planets that reside suspiciously close to a 4:2:1 period commensurability-as well as a few examples in the Kepler data set. Furthermore, it has been shown that multi-resonant states can serve as good candidates for the initial condition of the solar system (Morbidelli et al. 2007; Batygin \& Brown 2010).

In this section, we shall extend our analytical theory of the long-term dissipative evolution of resonant configurations to systems that comprise more than two planets. As will be shown below, the dynamics of multi-resonant systems can be quite rich in diversity, so for simplicity, we shall work with a system consisting of three planets, keeping in mind that extension to a larger number of resonant objects can be accomplished.

As above, let us begin by writing out the full Hamiltonian. The Keplerian part reads

$$
\mathcal{H}_{\mathrm{kep}}=-\frac{G^{2} M^{2} m_{1}^{3}}{2 \Lambda_{1}^{2}}-\frac{G^{2} M^{2} m_{2}^{3}}{2 \Lambda_{2}^{2}}-\frac{G^{2} M^{2} m_{3}^{3}}{2 \Lambda_{3}^{2}},
$$

while the resonant contribution is

$$
\begin{aligned}
\mathcal{H}_{\text {res }}= & -\frac{G^{2} M m_{1} m_{2}^{3}}{[\Lambda]_{2}^{2}}\left(f_{\text {res }}^{(1, \text { in })} \sqrt{\frac{2 \Gamma_{1}}{[\Lambda]_{1}}} \cos \left(\xi_{1}\right)\right. \\
& \left.+f_{\text {res }}^{(2, \text { in })} \sqrt{\frac{2 \Gamma_{2}}{[\Lambda]_{2}}} \cos \left(\xi_{2}^{\text {in }}\right)\right) \\
& -\frac{G^{2} M m_{2} m_{3}^{3}}{[\Lambda]_{3}^{2}}\left(f_{\text {res }}^{(1, \text { out })} \sqrt{\frac{2 \Gamma_{2}}{[\Lambda]_{2}}} \cos \left(\xi_{2}^{\text {out }}\right)\right. \\
& \left.+f_{\text {res }}^{(2, \text { out })} \sqrt{\frac{2 \Gamma_{3}}{[\Lambda]_{3}}} \cos \left(\xi_{3}\right)\right),
\end{aligned}
$$

where the four harmonics are

$$
\begin{aligned}
\xi_{1} & =k^{\text {in }} \lambda_{2}-\left(k^{\text {in }}-1\right) \lambda_{1}+\gamma_{1} \\
\xi_{2}^{\text {in }} & =k^{\text {in }} \lambda_{2}-\left(k^{\text {in }}-1\right) \lambda_{1}+\gamma_{2} \\
\xi_{2}^{\text {out }} & =k^{\text {out }} \lambda_{3}-\left(k^{\text {out }}-1\right) \lambda_{2}+\gamma_{2} \\
\xi_{3} & =k^{\text {out }} \lambda_{3}-\left(k^{\text {out }}-1\right) \lambda_{2}+\gamma_{3}
\end{aligned}
$$

and the superscripts "in" and "out" refer to the resonances of the inner and outer pair of planets, respectively. Before proceeding further, we note an important difference from the formalism developed in the previous section. In the two-planet case, dissipation caused both critical angles to collapse onto their respective foci. Let us examine if similar behavior is possible in the three planet case.

Suppose all four critical angles have evolved to a state where $d \xi / d t=0$. In this case, simultaneous zero-amplitude libration of $d \xi_{1} / d t-d \xi_{2}^{\text {in }} / d t=0$ and $d \xi_{2}^{\text {out }} / d t-d \xi_{3} / d t=0$ implies that the apses of the system are locked, i.e., $d \gamma_{1} / d t=$ $d \gamma_{2} / d t=d \gamma_{3} / d t=d \gamma_{\mathrm{sys}} / d t$. At the same time, expressing the mean longitude as $d \lambda / d t=n-d \gamma_{\text {sys }} / d t$, the relationship 
$d \phi_{2}^{\text {in }} / d t-d \phi_{2}^{\text {out }} / d t=0$ implies a strict correspondence among the semimajor axes: $-k^{\text {out }} n_{3}+\left(k^{\text {in }}+k^{\text {out }}-1\right) n_{2}-\left(k^{\text {in }}-1\right) n_{1}=0$. A configuration that obeys this relationship is in (or close to) nominal resonance (e.g., the Galilean satellites). This means that away from nominal resonance, only three out of four critical angles can reside at their respective foci, while the remaining angle will circulate with the frequency

$$
d \xi_{\text {circ }} / d t=-k^{\text {out }} n_{3}+\left(k^{\text {in }}+k^{\text {out }}-1\right) n_{2}-\left(k^{\text {in }}-1\right) n_{1} .
$$

Naturally, if the system is far from nominal resonance, then this circulation is comparatively fast, allowing us to drop (i.e., average over) the quickly varying harmonic and reduce the Hamiltonian (38) to a form that only contains three terms. This would further let us construct new action-angle coordinates, ensuring that the momenta conjugated to the three mean longitudes become constants of motion. However, identifying the circulating angle is not trivial a priori, since the calculation inevitably depends on the planetary physical parameters, and in some cases can have nonlinear dependence on initial conditions. Thus, unlike the two-planet problem described above, multi-resonant systems should be treated on a more case-bycase basis, as the construction of a suitable analytical theory for the long-term evolution depends on the properties of the system. Fortunately, as we already showed above, the timescale for the system to reach a quasi-stationary state is not much greater than the circularization timescale. So the initial transient period of system equilibration can be calculated numerically at a mild computational cost.

Due to the individual attention that multi-resonant planetary systems deserve, we shall leave the in-depth analysis of detected objects to follow-up papers and instead limit ourselves to an illustrative example of the long-term dynamical evolution of an equal-mass $\left(m_{1}=m_{2}=m_{3}=10^{-4} M\right)$ planetary system in a $4: 2: 1$ resonance. The aim of the calculation is largely to highlight the subtle differences between the evolution of a multi-resonant system and the results obtained for a single planetary pair in the previous sections.

With foresight, we begin with the construction of new canonically conjugated coordinates using the following generating function (intended for the system at hand):

$$
\begin{aligned}
F_{2}= & \lambda_{1} \Psi_{1}+\lambda_{2} \Psi_{2}+\lambda_{2} \Psi_{3}+\left(k^{\text {in }} \lambda_{2}-\left(k^{\text {in }}-1\right) \lambda_{1}+\gamma_{1}\right) \Phi_{1} \\
& +\left(k^{\text {out }} \lambda_{3}-\left(k^{\text {out }}-1\right) \lambda_{2}+\gamma_{2}\right) \Phi_{2} \\
& +\left(k^{\text {out }} \lambda_{3}-\left(k^{\text {out }}-1\right) \lambda_{2}+\gamma_{3}\right) \Phi_{3}
\end{aligned}
$$

which yields the variables

$$
\begin{aligned}
& \Psi_{1}=\Lambda_{1}+\left(k^{\text {in }}-1\right) \Phi_{1} \quad \psi_{1}=\lambda_{1} \\
& \Psi_{2}=\Lambda_{2}-k^{\text {in }} \Phi_{1}+\left(k^{\text {out }}-1\right)\left(\Phi_{2}+\Phi_{3}\right) \quad \psi_{2}=\lambda_{2} \\
& \Psi_{3}=\Lambda_{3}-k^{\text {out }}\left(\Phi_{2}+\Phi_{3}\right) \quad \psi_{3}=\lambda_{3} \\
& \Phi_{1}=\Gamma_{1} \quad \phi_{1}=k^{\text {in }} \lambda_{2}-\left(k^{\text {in }}-1\right) \lambda_{1}+\gamma_{1} \\
& \Phi_{2}=\Gamma_{1} \quad \phi_{2}=k^{\text {out }} \lambda_{3}-\left(k^{\text {out }}-1\right) \lambda_{2}+\gamma_{2} \\
& \Phi_{3}=\Gamma_{2} \quad \phi_{3}=k^{\text {out }} \lambda_{3}-\left(k^{\text {out }}-1\right) \lambda_{2}+\gamma_{3} .
\end{aligned}
$$

This choice of variables is appropriate when the angle $\xi_{2}^{\text {in }}$ is in circulation. Dropping this harmonic from the Hamiltonian renders $\left(\Psi_{1}, \Psi_{2}, \Psi_{3}\right)$ constants of motion (if instead, the circulating angle had been $\xi_{2}^{\text {out }}$, then the choice of $\Psi_{2}$ and $\phi_{2}$ would have been made as in Equation (13), identifying $k$ in Equation (13) with $k^{\text {in }}$, and the angle $\xi_{2}^{\text {out }}$ would have been dropped from the Hamiltonian).
After some manipulation (as described in the previous sections), the Hamiltonian takes on a simple form

$$
\begin{aligned}
\mathcal{H}= & \eta^{\text {in }} \Phi_{1}+\eta^{\text {out }} \Phi_{2}+\eta^{\text {out }} \Phi_{3}+\alpha^{\text {in }} \sqrt{2 \Phi_{1}} \cos \left(\phi_{1}\right) \\
& +\alpha^{\text {out }} \sqrt{2 \Phi_{2}} \cos \left(\phi_{2}\right)+\beta^{\text {out }} \sqrt{2 \Phi_{3}} \cos \left(\phi_{3}\right)
\end{aligned}
$$

where, as before,

$$
\begin{aligned}
& \eta^{\text {in }}=3\left([h]_{1}\left(k^{\text {in }}-1\right) \Psi_{1}-[h]_{2} k^{\text {in }} \Psi_{2}\right) \\
& \eta^{\text {out }}=3\left([h]_{2}\left(k^{\text {out }}-1\right) \Psi_{2}-[h]_{3} k^{\text {out }} \Psi_{3}\right)
\end{aligned}
$$

are the proximities to exact resonance. The coefficient $\alpha^{\text {in }}$ is given by Equation (20) and analogously,

$$
\begin{aligned}
\alpha^{\text {out }} & =-\frac{G^{2} M m_{2} m_{3}^{3}}{[\Lambda]_{3}^{2}} \frac{f_{\text {res }}^{(1, \text { out })}}{\sqrt{[\Lambda]_{2}}} \\
\beta^{\text {out }} & =-\frac{G^{2} M m_{2} m_{3}^{3}}{[\Lambda]_{3}^{2}} \frac{f_{\text {res }}^{(2, \text { out })}}{\sqrt{[\Lambda]_{3}}} .
\end{aligned}
$$

As shown in the previous section, under dissipation, the system will approach a quasi-stationary state. Once such a state is achieved, the corresponding fixed-point orbital parameters take on a familiar form

$$
\begin{aligned}
& e_{1} \rightarrow-\sqrt{\frac{1}{[\Lambda]_{1}} \frac{\alpha^{\text {in }}}{\eta^{\text {in }}}} \quad \phi_{1} \rightarrow \frac{1}{\eta^{\text {in }} \tau_{e_{1}}} \\
& e_{2} \rightarrow-\sqrt{\frac{1}{[\Lambda]_{2}} \frac{\alpha^{\text {out }}}{\eta^{\text {out }}}} \quad \phi_{2} \rightarrow \frac{1}{\eta^{\text {out }} \tau_{e_{2}}} \\
& e_{3} \rightarrow+\sqrt{\frac{1}{[\Lambda]_{3}} \frac{\beta^{\text {out }}}{\eta^{\text {out }}}} \quad \phi_{3} \rightarrow \pi-\frac{1}{\eta^{\text {out }} \tau_{e_{3}}} .
\end{aligned}
$$

It is important to recall that we have dropped a quickly varying resonant term from the Hamiltonian when deriving these equations. ${ }^{7}$ While the dropped harmonic will have little longlasting effect, it will act to introduce high-frequency "noise" into the solution, whose amplitude depends on the proximity of the system to exact three-body resonance. Thus, the equilibrium eccentricities and critical angles derived here are representative of average values.

Thus far, the behavior inferred from the above equations appears quite similar to the case of a single resonant pair described in the previous sections. However, an important difference surfaces when we consider the resonant drift of the semimajor axes:

$$
\begin{aligned}
\left(\frac{d \Lambda_{1}}{d t}\right)_{\text {res }}= & \frac{1-k^{\text {in }}}{\tau_{e_{1}}} \frac{\left(\alpha^{\text {in }}\right)^{2}}{\left(\eta^{\text {in }}\right)^{2}} \\
\left(\frac{d \Lambda_{2}}{d t}\right)_{\text {res }}= & \frac{k^{\text {in }}}{\tau_{e_{1}}} \frac{\left(\alpha^{\text {in }}\right)^{2}}{\left(\eta^{\text {in }}\right)^{2}}+\left(1-k^{\text {out }}\right) \\
& \times\left(\frac{1}{\tau_{e_{2}}} \frac{\left(\alpha^{\text {out }}\right)^{2}}{\left(\eta^{\text {out }}\right)^{2}}+\frac{1}{\tau_{e_{3}}} \frac{\left(\beta^{\text {out }}\right)^{2}}{\left(\eta^{\text {out }}\right)^{2}}\right) \\
\left(\frac{d \Lambda_{3}}{d t}\right)_{\text {res }}= & k_{\text {out }}\left(\frac{1}{\tau_{e_{2}}} \frac{\left(\alpha^{\text {out }}\right)^{2}}{\left(\eta^{\text {out }}\right)^{2}}+\frac{1}{\tau_{e_{3}}} \frac{\left(\beta^{\text {out }}\right)^{2}}{\left(\eta^{\text {out }}\right)^{2}}\right) .
\end{aligned}
$$

\footnotetext{
7 Had the quickly varying harmonic been $\xi_{2}^{\text {out }}$ instead of $\xi_{2}^{\text {in }}$, the coefficients in front of terms containing $\Phi_{2}$ in Equation (43) would have been $\beta^{\text {in }}$. Equation (46) would then be modified accordingly.
} 

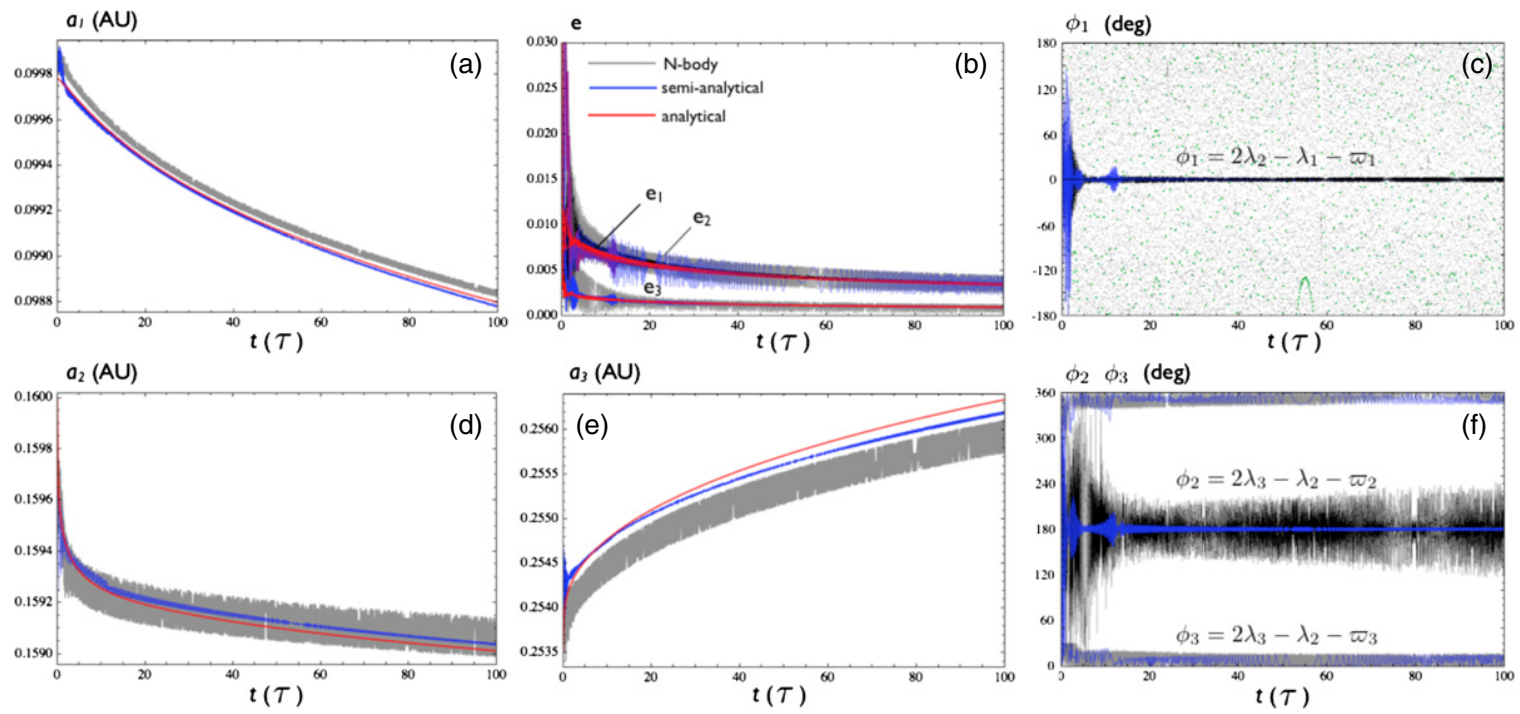

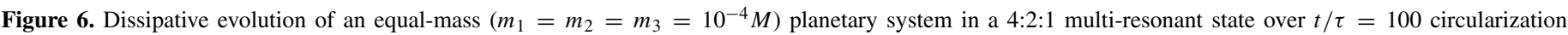

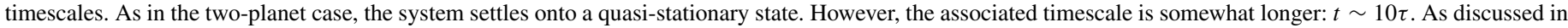

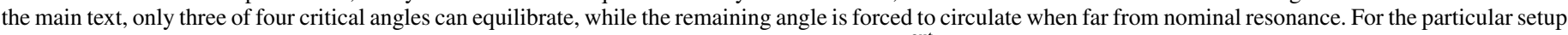

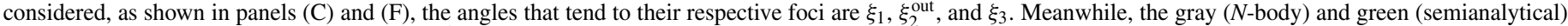

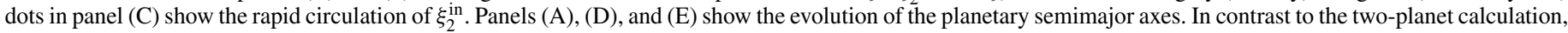

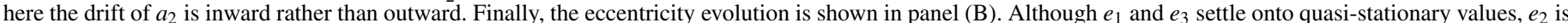

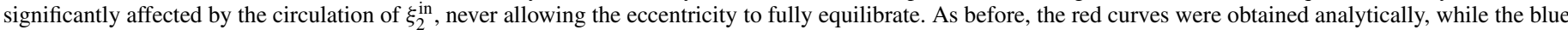

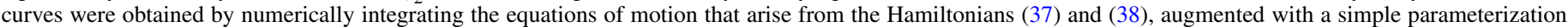

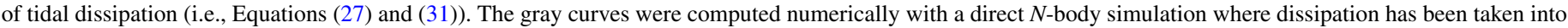
account. Note the excellent quantitative agreement between the theory and the numerics.

(A color version of this figure is available in the online journal.)

As in the two-planet case, the drifts of the innermost and outermost planets are inward and outward, respectively. The migration direction of the second planet, however, depends on the relative strengths of the inner and outer resonances, since the first term is positive definite while the second term is negative definite. Indeed, one could envision a set of system parameters (e.g., $m_{3} \ll m_{2}, m_{1}$ ) where tidal dissipation leads to a divergence away from one set of resonances (increasing $\left|\eta^{\text {in }}\right|$ ) and convergence onto another set of resonances (decreasing $\left.\left|\eta^{\text {out }}\right|\right)$. In the context of such a scenario, conservation of the null phase-space area occupied by a quasi-stationary orbit will lead to eccentricity growth (this can be inferred from Equation (46)). At the same time, it is important to recall that the equations presented were derived as an expansion around the nominal resonance location (which is assumed constant), and thus require dissipation in order to give rise to the corresponding drift of the semimajor axes. That is, one could in principle envision a scenario where only $\tau_{e_{1}}$ is finite, for which Equation (47) would predict a diverging inner pair and a stationary outermost planet, inconsistent with resonant capture (and the associated drift of the nominal resonance location, $d[\Lambda] / d t)$. However, as already pointed out above, the resonant harmonics are nonlinearly coupled. Consequently, such a situation is atypical in practice, since dissipation on a single planet also results in damping of the other planet's eccentricities.

The application of the developed theory is demonstrated in Figure 6. For the particular illustrative setup considered here, the angles $\left(\phi_{1}, \phi_{2}, \phi_{3}\right)$ attain a near-focal state within $t \sim 10 \tau$ while the dropped harmonic continues its circulation as expected. Although all three eccentricities decay monotonically as before, there is a clear qualitative difference in the behavior of $e_{2}$ compared to that of the two-planet case. In particular, $e_{2}$ never settles onto a fixed point and is instead continuously driven by the circulation of $\xi_{2}^{\text {in }}$, which contains $\gamma_{2}$, an angle conjugated to $\Gamma_{2} \propto e_{2}^{2}$. Perhaps unsurprisingly, $e_{1}$ and $e_{3}$ are not strongly affected by this circulation.

A more important distinction between the two-planet and three-planet evolutions is the direction of the second planet's drift. Namely, the combined effect of tidal dissipation and resonant interactions is now to drive the middle planet inward, whereas the evolution of $a_{2}$ was positive definite in the twoplanet case. All of this hints at the wide variety of possible outcomes and the dynamical richness of the multi-resonant interactions in the presence of dissipative forces.

\section{DISCUSSION}

The primary aim of this work has been to formulate a simple, physically intuitive analytical theory for the dissipative divergence of resonant orbits. We began with a purely conservative treatment of a single resonant pair and showed that at sufficiently low eccentricities and limited libration amplitudes, resonant dynamics can be treated with a linear, integrable approximation to the full resonant Hamiltonian. We then introduced simply parameterized tidal dissipation into the equations of motion and showed that the system tends to a quasi-stationary state over a few eccentricity circularization timescales. The collapse of the critical angles onto near-focal values in turn results in a divergent drift of the semimajor axes such that the outer orbit continually gains orbital energy while the inner planet's orbit decays. We subsequently showed how the developed formalism can be extended to multi-resonant systems. However, we have limited ourselves to a single illustrative example of the evolution of a system near a Laplace-like resonance, as we argued that the parameter space available to multi-resonant systems is quite large, rendering individual modeling more cost effective.

Overall, our results point at the distinct possibility that the dynamical architectures of numerous detected systems, 
whose orbits seem to lie outside of resonance on the basis of the observed orbital periods, are a result of resonantly aided dissipative divergence of the orbits (Papaloizou 2011), and thus comprise a number of important implications. First, the explanation we propose suggests that protoplanetary disks are indeed conducive to forming resonant planetary systems, whose long-term survival is assured (Cresswell \& Nelson 2008). In combination with precise quantitative modeling, this constraint can likely yield important new insights into understanding the physical structure and evolution of protoplanetary disks (e.g., weakly turbulent).

Second, as shown in Section 3, depending on the mass ratio and the elapsed time, resonant orbits can evolve up to tens of percent away from nominal resonance. If such extreme evolution is common, then it is possible that many planetary systems are actually in resonance even if their orbital periods are apparently not in commensurability. In particular, we expect the periodratio statistics of newly formed planetary systems to cluster more clearly around resonant values than those of an evolved sample (see Fabrycky et al. 2012 for an in-depth discussion of the current data set).

Third, the fact that the time dependence of the orbital divergence is related to the tidal circularization timescale can be used to infer from the observed period ratio how many circularization timescales a given system has evolved through, if the age of the system is known. Such information is vital for constraining unobservable parameters of extrasolar planetary systems such as the planetary tidal quality factor (Goldreich \& Soter 1966), whose origin remains largely unexplained and is among the most poorly constrained values in astrophysics. Although the above arguments hinge on the observationally elusive characterization of the physical planetary properties, we can certainly expect the data to improve continuously over the coming years, allowing for these calculations to be executed, eventually.

We thank Kleomenis Tsiganis, Peter Goldreich, and Greg Laughlin for numerous useful conversations. During the preparation of this paper, we have become aware that Y. Lithwick \& Y. Wu (2012) arrived at similar arguments simultaneously and independently. K. Batygin acknowledges supported from NASA's NESSF graduate fellowship.

\section{REFERENCES}

Adams, F. C., Laughlin, G., \& Bloch, A. M. 2008, ApJ, 683, 1117

Balbus, S. A., \& Hawley, J. F. 1991, ApJ, 376, 214

Batygin, K., \& Brown, M. E. 2010, ApJ, 716, 1323

Batygin, K., \& Laughlin, G. 2011, ApJ, 730, 95

Batygin, K., \& Morbidelli, A. 2011, Celest. Mech. Dyn. Astron., 111, 219

Bodenheimer, P., Lin, D. N. C., \& Mardling, R. A. 2001, ApJ, 548, 466

Borucki, W. J., Koch, D. G., Basri, G., et al. 2011, ApJ, 728, 117

Chambers, J. E. 1999, MNRAS, 304, 793

Chirikov, B. V. 1979, Phys. Rep., 52, 263

Cresswell, P., \& Nelson, R. P. 2008, A\&A, 482, 677

Delisle, J.-B., Laskar, J., Correia, A. C. M., \& Boué, G. 2012, arXiv:1207.3171

Eggleton, P. P., Kiseleva, L. G., \& Hut, P. 1998, ApJ, 499, 853

Fabrycky, D. C., Lissauer, J. J., Ragozzine, D., et al. 2012, arXiv:1202.6328

Goldreich, P. 1965, MNRAS, 130, 159

Goldreich, P., \& Soter, S. 1966, Icarus, 5, 375

Henrard, J., \& Lamaitre, A. 1983, Celest. Mech., 30, 197

Ketchum, J. A., Adams, F. C., \& Bloch, A. M. 2011, ApJ, 726, 53

Lee, M. H., \& Peale, S. J. 2002, ApJ, 567, 596

Lissauer, J. J., Ragozzine, D., Fabrycky, D. C., et al. 2011, ApJS, 197, 8

Lithwick, Y., \& Wu, Y. 2012, arXiv:1204.2555

Mardling, R. A. 2007, MNRAS, 382, 1768

Mardling, R. A., \& Lin, D. N. C. 2002, ApJ, 573, 829

Mayor, M., Udry, S., Lovis, C., et al. 2009, A\&A, 493, 639

Message, P. J. 1966, in IAU Symp. 25, The Theory of Orbits in the Solar System and in Stellar Systems, ed. G. I. Kontopoulos (London: Academic press), 197

Morbidelli, A. (ed.) 2002, Modern Celestial Mechanics: Aspects of Solar System Dynamics (London: Taylor and Francis)

Morbidelli, A., Tsiganis, K., Crida, A., Levison, H. F., \& Gomes, R. 2007, AJ, 134,1790

Murray, C. D., \& Dermott, S. F. 1999, in Solar System Dynamics, ed. C. D. Murray (Cambridge: Cambridge Univ. Press)

Papaloizou, J. C. B. 2011, Celest. Mech. Dyn. Astron., 111, 83

Papaloizou, J. C. B., \& Terquem, C. 2010, MNRAS, 405, 573

Peale, S. J. 1976, ARA\&A, 14, 215

Peale, S. J. 1986, in Satellites, ed. J. A. Burns \& M. S. Matthews (Tucson, AZ: Univ. Arizona Press), 159

Press, W. H., Teukolsky, S. A., Vetterling, W. T., \& Flannery, B. P. 1992, Numerical Recipes in FORTRAN. The Art of Scientific Computing (2nd ed.; Cambridge: Cambridge Univ. Press)

Rein, H., \& Papaloizou, J. C. B. 2009, A\&A, 497, 595

Rivera, E. J., Laughlin, G., Butler, R. P., et al. 2010, ApJ, 719, 890

Rogers, L. A., Bodenheimer, P., Lissauer, J. J., \& Seager, S. 2011, ApJ, 738,59

Stevenson, D. J. 1982, Annu. Rev. Earth Planet. Sci., 10, 257

Terquem, C., \& Papaloizou, J. C. B. 2007, ApJ, 654, 1110

Wisdom, J. 1980, AJ, 85, 1122

Wu, Y., \& Goldreich, P. 2002, ApJ, 564, 1024 